\title{
CHAPTER 6 \\ The role of bacterial adhesion to epithelial cells in pathogenesis
}

\section{INTRODUCTION}

Colonizing host epithelia represents a formidable challenge to bacterial pathogens. To a large extent, epithelial surfaces are designed to shield the multicellular organism from the environment and to protect the body interior from potentially harmful microbes. Where epithelial surfaces permit exchange of molecular components with the external world, a multitude of innate and acquired host defence mechanisms keep microorganisms in check. In addition, invading pathogens either have to compete successfully with resident commensal bacteria for space and nutrients or have to reach and establish themselves in otherwise sterile parts of the body. As we will discuss in this chapter, the specific interaction with host surface components and the tight adhesion to epithelial cells form one of the common ways in which bacterial pathogens have evolved to successfully accomplish the colonization of their respective host organism. It is important to emphasize that in many cases, this initial host-microbe encounter at the epithelial barrier is not only a critical determinant of pathogen-host specificity and range but also, to a large extent, a decisive point for the infection process as a whole.

Adhesion of pathogenic bacteria to host cells had been observed at the beginning of the twentieth century in the early days of investigations into the microbiological origin of infectious diseases (Guyot, 1908). However, the concept that bacterial adhesion to host surfaces often represents an essential step in the development of infection matured only several decades later (Beachey, 1981; Duguid, 1959; Eden et al., 1976; McNeish et al., 1975; Punsalang and Sawyer, 1973). Mechanical cleansing processes, ranging from eye-blinking to

Konstanzer Online-Publikations-System (KOPS) URL: http://www.ub.uni-konstanz.de/kops/volltexte/2007/4269/ URN: http://nbn-resolving.de/urn:nbn:de:bsz:352-opus-42691 
the flushing of the urethra and the constant turnover of the mucous layer will remove any non-attached particles from the epithelium. Contacting the host surface and establishing a dependable adhesive connection is, therefore, key to successful colonization. The elucidation of adhesion components and the ability to genetically manipulate these components on both the pathogen and the host side have underscored the critical importance of bacterial adhesion in infectious diseases. In several models of bacterial infection, the molecular nature of the adhesin and the respective host receptors is now well documented. Although other bacterial surface structures, such as lipopolysaccharide, wall teichoic acid, and lipoteichoic acid, can contribute to cell adhesion (Edwards et al., 2000; Ofek et al., 1975; Paradis et al., 1999; Weidenmaier et al., 2004), the main bacterial adhesins characterized so far are proteins.

\section{FIMBRIAL ADHESINS}

In a broad sense, adhesive bacterial proteins can be classified into fimbrial and non-fimbrial (or afimbrial) adhesins. As a rule, fimbriae are long $(>1 \mu \mathrm{m})$ and usually thin $(2-8 \mathrm{~nm})$ protrusions of the bacterial surface that are sometimes referred to as pili. The term "pilus" was originally used to describe bacterial organelles involved in the conjugative transfer of DNA, but it is now also applied regularly to adhesive surface structures. Fimbrial protrusions are made up of major (with more than 100 copies per pilus) and minor protein subunits that often, but not always, carry out the structural and the adhesive functions, respectively, of these microbial organelles. Classical examples of fimbrial adhesins are found in the Pap and Fim systems of uropathogenic Escherichia coli (UPEC) and the type IV pili of Pseudomonas aeruginosa, enteropathogenic E. coli, and pathogenic Neisseriae; the molecular components and the roles of these fimbriae for epithelial cell adhesion have been summarized in several reviews (Craig et al., 2004; Jonson et al., 2005; Kau et al., 2005; Sauer et al., 2004).

It appears that the long surface protrusions are an evolutionarily optimized tool to establish the initial bacterial contact with the epithelial barrier. Indeed, the thin and bendable fimbriae are ideally suited for the task of longrange contact, as they help to circumvent the electrostatic repulsion forces between the two negatively charged surfaces of the eukaryotic cell and the microbe. Moreover, at least type IV pili also seem to work in a manner similar to grappling hooks, as the pilus fiber can be retracted by the bacterium and thereby exert force (Maier et al., 2002). This fascinating dynamic nature of the pilus not only can confer motility to single suspended bacteria (Merz 
Table 6.1 Fimbrial adhesins and recognized host cell structures

\begin{tabular}{lll}
\hline \hline Species & Adhesin & Recognized structure \\
\hline Escherichia coli & FimH & Mannose \\
& FaeG & Gaß1-3Gal \\
& GafD & N-acetyl-D-glucosamine \\
& PapG & Galß1-4Gal \\
& SfaS & NeuAc $\beta 2-3 G a l \beta 1-3 G a l N a c$ \\
& CfaB & NeuAc-GM2 \\
& G-Fimbrien & GlcNAc \\
& TypIV pili & CD46 (?) \\
Neisseria gonorrhoeae & TypIV pili & L-Fucose \\
Vibrio cholerae & & \\
\hline \hline
\end{tabular}

et al., 2000) but also endows the microbes with a means to actively narrow the gap between the piliated bacterium and the tissue surface. The result of pilus retraction in cell-associated bacteria, therefore, is a close apposition between the two membranes, allowing additional short-reach interactions to take place. An overview of fimbrial adhesins from different pathogenic microorganisms and their host receptors is given in Table 6.1.

As can be seen in Table 6.1, fimbrial adhesins most often function as lectins and recognize carbohydrate moieties found on membrane-embedded glycoproteins and glycolipids. Accordingly, low-complexity carbohydrates have the ability to interfere with the adhesion of bacterial pathogens, a phenomenon recognized well before the molecular characterization of the respective adhesins (Old, 1972). However, so far it has not been possible to translate these findings into novel clinical treatment options that prohibit bacterial attachment to epithelial cells and thereby block bacterial infections right from the start (Bouckaert et al., 2005).

\section{AFIMBRIAL ADHESINS}

In addition to fimbrial adhesins, a large variety of non-fimbrial adhesins has been characterized. As the name implies, these adhesins are either embedded into or attached to the outer surface of the microbe and are usually not localized on surface protrusions. As we will see in the following examples, this type of adhesin often engages in direct protein-protein interactions with host components. Non-fimbrial adhesins are found in a variety of Gram-negative bacteria, but they are also common in Gram-positive 
bacteria, where few fimbrial adhesins have been described so far (Ton-That and Schneewind, 2004). Examples of non-fimbrial adhesins from Grampositive microbes include internalin A (InlA) and InlB from Listeria monocytogenes. Similar to many other adhesins, these two proteins not only mediate tight binding of the bacteria to host cells but also trigger, via their recognized cellular receptors, actin cytoskeleton rearrangements, leading to bacterial internalization (Pizarro-Cerda et al., 2004).

The initial InlA-mediated interaction of L. monocytogenes with intestinal epithelial cells is a prominent example of the essential role that adhesion to epithelia often plays in bacterial pathogenesis. Guinea pig and human intestinal epithelial cells allow InlA-dependent attachment of L. monocytogenes; this is due to the presence of a highly homologous E-cadherin, the cellular receptor for InlA, in these two mammalian species (Mengaud et al., 1996). In contrast to humans and guinea pigs, mice are very resilient to oral infection, the normal entry site of food contaminating L. monocytogenes; this resistance could be attributed by a series of elegant experiments to a single amino-acid difference in mouse E-cadherin (glutamine at position 16) compared with human or guinea pig E-cadherin (proline at position 16) (Lecuit et al., 1999). Moreover, when mice are engineered to express human E-cadherin in their intestines, these animals now become highly susceptible to oral infection by L. monocytogenes (Lecuit et al., 2001).

The atomic structure of the adhesin-receptor complex has further illuminated the intricate handshake-like protein-protein interaction between InlA and E-cadherin (Schubert et al., 2002). This submolecular view has also underpinned the critical position of Pro-16 in the center of the InlA-E-cadherin interface (Schubert et al., 2002). The elucidation of InlA binding specificity and the molecular details of InlA-E-cadherin interaction have highlighted the essential role of this interaction in the establishment of the disease. Together, these investigations provide us with a marvelous example of how host specificity can be determined by bacterial adhesin-host receptor pairs.

\section{INTEGRIN-BINDING AFIMBRIAL ADHESINS OF STAPHYLOCOCCI}

Other Gram-positive bacteria, such as Staphylococcus aureus and Streptococcus pyogenes, have evolved a number of surface proteins that bind to serum and matrix components of their host (Foster and Hook, 1998; Patti et al., 1994). These types of afimbrial adhesin include fibronectin-binding protein A and B (FnBP-A and -B) of S. aureus and streptococcal fibronectinbinding protein 1 (Sfb1) (also termed F1) of S. pyogenes. Both, FnBPs and Sfb1 
attach the plasma component and extracellular matrix protein fibronectin to the surface of the bacteria by an intriguing tandem beta-zipper mechanism (Schwarz-Linek et al., 2003).

It is thought that binding to extracellular matrix proteins such as fibronectin allows the bacteria to colonize matrix-coated surfaces such as implanted medical devices. Furthermore, the bacterial adhesin does not only mediate direct binding to fibronectin-covered surfaces but also utilizes fibronectin as a molecular bridge, indirectly linking the bacterial surface with the principal host fibronectin receptor, the integrin $\alpha_{5} \beta_{1}$ (Ozeri et al., 1998; Sinha et al., 1999). Upon bacterial adhesion, integrin ligation can lead to efficient internalization of the bacteria into eukaryotic cells in vitro and in vivo (Agerer et al., 2003, 2005; Brouillette et al., 2003; Ozeri et al., 1998; Sinha et al., 1999). Interference with fibronectin binding to the bacteria by either genetic deletion of FnBP proteins or administration of a recombinant fibronectin binding domain of FnBP as a competitive inhibitor during infections can attenuate staphylococcal virulence in several disease models, such as abscess formation, mastitis, and endocarditis (Brouillette et al., 2003; Kuypers and Proctor, 1989; Menzies et al., 2002).

However, the role of FnBPs in staphylococcal infections is not clear-cut, and results from experimental models have been reported where FnBPs did not contribute to the virulence of this pathogen (for review, see (Menzies, 2003). As staphylococci are associated with a large variety of clinical manifestations, often involving secreted toxins, it is very likely that cell adhesion and, in particular, FnBPs will act as bona fide virulence factors only in some of these situations. It is also conceivable that such FnBP-mediated adhesion and invasion processes are not only found in acute disease settings but also are of importance for the persistence of staphylococci in their host. Together, current experimental and epidemiologic data support the view that FnBPs contribute in some settings to the virulence of $S$. aureus and that cellular invasion via integrins represents one of the functional properties conferred by FnBP expression.

\section{STAPHYLOCOCCAL INTERNALIZATION VIA MATRIX-BINDING INTEGRINS}

Interestingly, most integrins usually operate in the context of immobilized matrix proteins and are not considered to mediate endocytosis of attached ligands. In particular, integrin $\alpha_{5} \beta_{1}$, the fibronectin receptor, has been implied in organizing extracellular fibronectin into a fibrillar network by 
exerting force on the immobilized matrix protein (Schwarzbauer and Sechler, 1999). It is interesting to speculate that in contrast to immobilized fibronectin, the integrin-attached bacteria are pulled into the cell by the same cellular force-generating machinery that is used to remodel the fibronectin matrix under physiologic conditions.

In general, extracellular matrix contact and integrin ligation induce the formation of protein complexes at the cytoplasmic aspect of cell adhesion sites. These protein complexes have been termed focal adhesions or focal contacts, as they occur at discrete focal spots along the matrix-facing surface of the adherent eukaryotic cell. Focal complexes have important structural and signaling functions, as they dynamically link the clustered and ligand-bound integrins to the intracellular actin network that, together with myosin, is responsible for force generation (Zaidel-Bar et al., 2004). Due to their morphologic appearance and their functional connection to fibronectin fibril assembly, integrin $\alpha_{5} \beta_{1}$-initiated protein complexes have been termed fibrillar adhesions (Zamir and Geiger, 2001). A characteristic component of such adhesive structures is tensin, an actin-binding adaptor molecule (Zamir et al., 2000). In addition, signaling enzymes, such as the protein tyrosine kinases Src and focal adhesion kinase (FAK), have been implied in the generation of fibrillar adhesions and the integrin $\alpha_{5} \beta_{1}$-mediated assembly of a fibrillar fibronectin network (Ilic et al., 2004; Volberg et al., 2001).

Work has now started to address the role of these integrin-associated host cell factors for the FnBP-mediated attachment and invasion of S. aureus. Engagement of integrin $\alpha_{5} \beta_{1}$ by fibronectin-binding staphylococci indeed induces the formation of fibrillar adhesion-like protein complexes at the site of bacterial attachment, as characterized by the recruitment of tensin, FAK, zyxin, and vinculin (Agerer et al., 2005). Furthermore, interference with Src or FAK function abrogates the internalization of the bacteria via integrin $\alpha_{5} \beta_{1}$ and suppresses the increased tyrosine phosphorylation observed at bacterial attachment sites (Agerer et al., 2003, 2005). One of the effectors of activated FAK and Src kinases during integrin-mediated internalization has been identified as cortactin, an actin-binding protein that can associate with the Arp2/3 complex and promote actin polymerization but that can also bind to dynamin-2, a regulator of membrane endocytosis (McNiven et al., 2000; Selbach and Backert, 2005). Together, these investigations support the view that fibronectin-coated staphylococci induce fibrillar adhesion-like contact sites that are regulated by protein tyrosine kinase signaling and link the bacteriaoccupied integrins with the intracellular actin cytoskeleton. It is tempting to speculate that in this case bacterial internalization is promoted not only by increased actin polymerization generating membrane protrusions that 
enclose the pathogen but also, at least partially, by the contraction forces generated by the integrin-connected intracellular actin-myosin network that, under physiological conditions, promotes fibronectin fibrillogenesis.

\section{INTEGRIN ENGAGEMENT BY ENTEROPATHOGENIC YERSINIAE}

In contrast to the indirect way in which staphylococci and streptococci exploit host integrin $\alpha_{5} \beta_{1}$, the enteropathogenic Yersinia species, Y. enterocolitica and Y. pseudotuberculosis, bind directly to $\beta_{1}$-containing integrins and thereby trigger uptake by host cells. Because of its invasive property, the bacterial adhesin responsible for integrin binding and cellular internalization has been coined invasin; it serves as the prototype for this class of proteins (Isberg and Leong, 1990). Latex beads coated with invasin or non-pathogenic E. coli expressing invasin are taken up efficiently by different cell types, demonstrating that this protein is sufficient to confer invasiveness (Dersch and Isberg, 1999; Isberg and Leong, 1990). Since its discovery, several seminal contributions have illuminated the structural determinants of invasin required for integrin binding as well as the host factors required to allow invasin-initiated uptake (see review in Isberg et al., 2000). It is interesting to note that the Cterminal domains of this bacterial surface protein seem to mimic fibronectin type III repeats 9 and 10 that are involved in integrin binding. Indeed, invasin can competitively inhibit fibronectin association with integrin $\alpha_{5} \beta_{1}$ demonstrating that these two proteins bind to the same site in integrins (Isberg et al., 2000).

Invasin expression seems to allow the orally ingested Yersinia to overcome the intestinal barrier. Indeed, invasin-deficient bacteria are impaired in their ability to transverse the intestinal lining (Marra and Isberg, 1997). In this case, it is thought that invasin-expressing Yersinia do not attach to the regular enterocytes, polarized epithelial cells that do not expose integrins on their apical surface. However, a specialized subset of intestinal epithelial cells, the so-called microfold or M-cells, does expose integrins to the gut lumen (Clark et al., 1998). In this context, it is also worth mentioning that a close relative of these pathogens, the plague bacterium Yersinia pestis, does not express invasin. The respective coding sequence is found in the genome, and yet it is inactivated by the insertion of an insertion sequence (IS) element (Simonet et al., 1996). As Y. pestis is transmitted by an arthropod vector directly into the bloodstream of the host, these bacteria do not have to overcome an epithelial barrier and, therefore, invasin expression might not be required any more. 
Table 6.2 Pathogens targeting extracellular matrix (ECM)-binding integrins

\begin{tabular}{ll}
\hline \hline Species & ECM protein/integrin subunit \\
\hline Borrelia burgdorferi & $\mathrm{FN} / \beta 1$ integrins \\
Mycobacterium leprae & $\mathrm{FN}, \mathrm{LN} / \beta 1$, and $\beta 4$ integrins \\
Mycobacterium bovis BCG & $\mathrm{FN} / \beta 1$ integrins \\
Neisseria gonorrhoeae and $N$. meningitidis & $\mathrm{FN}, \mathrm{VN} / \beta 1$, and $\beta 3$ integrins \\
Porphyromonas gingivalis & $\beta 1$ integrins \\
Shigella flexneri & $\beta 1$ integrins \\
Staphylococcus aureus & $\mathrm{FN}, \mathrm{LN}, \mathrm{Col} / \beta 1$ integrins \\
Streptococcus pyogenes and $S . d y$ sgalactiae & $\mathrm{FN} / \beta 1$ integrins \\
Yersinia pseudotuberculosis and Y. enterocolitica & $\beta \times 1$ integrins \\
\hline \hline
\end{tabular}

Col, collagen; FN, fibronectin; LN, laminin; VN, vitronectin.

Direct or indirect engagement of matrix-binding integrins is also observed for other pathogenic bacteria (listed in Table 6.2). Although integrins are exploited by multiple bacterial and viral pathogens as well as parasites, it is not straightforward to conceive the role of this interaction in the context of an intact epithelium. In particular, integrins are distributed on the basolateral side of polarized epithelial cells, which would be inaccessible for bacteria colonizing the throat, intestine, or urogenital tract. Therefore, it has been speculated that these types of adhesin come into play only after the initial contact of the microbes with the tissue surface. As shown in several examples, pathogenic bacteria can influence the integrity of the epithelial barrier either directly by secreted toxins or indirectly by inducing granulocyte influx and tissue destruction (McCormick, 2003). Damage to the epithelial lining might then allow microbes to gain access to basolateral components such as integrins or cadherins.

\section{COOPERATION BETWEEN FIMBRIAL AND AFIMBRIAL ADHESINS: THE PARADIGM OF PATHOGENIC NEISSERIAE}

Fimbrial and afimbrial adhesins are not mutually exclusive, but often they are expressed simultaneously on the same microorganism. The pathogenic Neisseriae are one of the well-characterized examples, where both types of adhesin seem to act in concert and together coordinate a multistep and complex process of cell attachment. The remainder of this chapter 
concentrates on this paradigm in order to highlight some of the features of bacterial adhesins and receptor targeting. Furthermore, in light of advances, this overview is extended beyond the direct adhesin-receptor interaction to cover some of the specific responses of the host epithelial cell following bacterial binding.

Both pathogenic neisserial species, $N$. gonorrhoeae and $N$. meningitidis (the gonococcus and the meningococcus, respectively), are highly specialized colonizers of the human mucosa. Gonococci and meningococci are known to express fimbriae; the presence of these adhesive surface structures is subject to phase variation. Fimbriated Neisseriae have a high tendency to stick together in microcolonies, as the pilus supports bacteria-bacteria adhesion. Moreover, it is generally accepted that type IV pilus-mediated interactions are the initial event allowing both unencapsulated $N$. gonorrhoeae and encapsulated meningococci to colonize the epithelial surface of the urogenital tract or the nasopharynx, respectively (Kellogg et al., 1963; Punsalang and Sawyer, 1973), although direct in vivo proof for this concept is still lacking (Cohen and Cannon, 1999). In vitro, piliated Neisseria adhere avidly to polarized human epithelial cells and form microcolonies on the apical surface (Pujol et al., 1997). These microcolonies are due to bacterial replication but also seem to arise from pilus-initiated bacterial aggregates that attach to the cell surface. The PilX protein has been identified as a factor promoting bacterial aggregation in the presence of pili (Helaine et al., 2005). Deletion of the PilX encoding gene not only disrupts bacterial aggregates but also dramatically reduces attachment to host cells (Helaine et al., 2005). These data support the view that pilus-mediated cell attachment requires prior bacteria-bacteria binding and suggests that bacterial aggregates allow bacterial adhesion even in the case of low-affinity interaction between the bacterial adhesin and the cellular receptor by increasing avidity. This would also be in line with the obvious difficulty in unequivocally identifying a cellular receptor for the neisserial pilus, as such a low-affinity interaction might be hard to detect by standard biochemical approaches.

The main subunit of the gonococcal type IV pilus, pilin encoded by the pilE gene, is a textbook paradigm for its astounding antigenic variability. Antigenic variation of pilin is generated by a recombination-based exchange of coding sequences between promoter-less silent gene loci and actively expressed PilE loci (Meyer et al., 1990). Although pilin can agglutinate erythrocytes, it has always been questioned whether the variable PilE can encode the principal adhesin of the neisserial type IV pilus. Work from several laboratories has suggested that a minor pilus subunit, the PilC protein encoded by two alleles in the genome, is the adhesive factor (Morand 
et al., 2001; Rudel et al., 1995). Indeed, purified gonococcal PilC binds to human cells and inhibits adhesion of piliated N. gonorrhoeae and N. meningitidis (Scheuerpflug et al., 1999). PilC-deficient bacteria are impaired in their adhesion to human epithelial cells but also seem to be compromised in their capacity for natural transformation and motility, both pilus-dependent traits (Morand et al., 2004; Ryll et al., 1997), suggesting that PilC could have multiple functions. At least in meningococci it appears that only one of the two pilC alleles, pilC1, entails adhesive properties (Morand et al., 2001). So far, no binding partner for the PilC adhesin on human cells has been described (Kirchner and Meyer, 2005). However, work from the group of Jonsson has suggested that human CD46 serves as a cellular receptor for pilitated Neisseria on epithelial cell lines (Kallstrom et al., 1997). In other situations, a strict correlation between CD46 expression and pilus-mediated adhesion of Neisseria has not been detected (Tobiason and Seifert, 2001), and CD46-mediated binding of piliated gonococci has not been observed in other studies (Kirchner et al., 2005). Interestingly, although mice transgenic for human CD46 show a higher mortality rate after intraperitoneal injection of meningococci, the virulence of the bacteria in this in vivo model is independent of a piliated phenotype (Johansson et al., 2003).

Despite the still debated role of CD46 as a cellular receptor for pilusdependent interactions, it is clear that pilus-mediated attachment to epithelial cells evokes a number of host responses, ranging from changes in cytosolic $\mathrm{Ca}^{2+}$ levels to tyrosine phosphorylation of host proteins (Ayala et al., 2001; Hoffmann et al., 2001; Kallstrom et al., 1998; Lee et al., 2002). These cellular events are observed within minutes to a few hours after infection, coinciding with the initial local adherence of the microbes, when bacterial microcolonies are found on top of the cells. However, several hours after the pilusmediated contact, pilus and capsule expression are downregulated in the case of meningococci (Deghmane et al., 2002), suggesting that the bacteria sense the presence of, or the attachment to, the eukaryotic cells by a currently unknown mechanism. As pilus expression ceases, the pattern of adhesion is altered, indicating that another type of adhesive contact is established. By scanning electron microscopy, it has been observed that the bacterial microcolonies are resolved, and singly attached bacteria are found distributed over the epithelial cell surface (Pujol et al., 1997). This form of attachment has been termed "diffuse adherence" and seems to represent a further step in the microbe-host cell interaction.

It is thought that at this point a second group of neisserial adhesins, the colony opacity-associated (Opa) proteins, come into play. In both pathogenic neisserial species, multiple functional copies of opa genes are distributed over 
the entire genome. Transcription of Opa proteins is constitutive; however, expression undergoes a high rate of phase variation by a translation-based mechanism (Stern and Meyer, 1987; Stern et al., 1986). Therefore, a natural population of these pathogens will comprise both non-opaque and opaque organisms, with some having a single Opa protein but others expressing multiple Opas at a given time. As there are up to 12 copies of opa genes in the gonococcal genome, the possibilities for variation are enormous. It is interesting to note that in human-volunteer challenge experiments, an initial inoculum of non-opaque gonococci can establish disease at infectious doses comparable with opaque phenotypes. However, most of the bacteria that are reisolated from the infected volunteers have switched to an opaque phenotype (Jerse et al., 1994; Swanson et al., 1988). These results suggest that Opa proteins, although not essential for the initial contact with the host, play a beneficial role for the pathogen during colonization and multiplication in vivo.

In contrast to the bacterial type IV pilus, Opa proteins are embedded within the outer bacterial membrane and therefore belong to the group of afimbrial adhesins. Secondary structure predictions suggest that mature Opa proteins possess eight membrane-spanning domains arranged as antiparallel $\beta$-strands, giving rise to a membrane-embedded $\beta$-barrel with four extracellular loops (Bhat et al., 1991; de Jonge et al., 2002; Malorny et al., 1998). Although the crystal structure of Opa proteins is currently unknown, the structure of the related NspA protein from N. meningitidis has been solved and suggests that the extracellular loops constitute a conformational binding interface (Vandeputte-Rutten et al., 2003). On the basis of sequence comparison of multiple gonococcal and meningococcal Opa proteins, the amino-acid sequence of the central two extracellular loops has been found to be hypervariable, and they have been termed hypervariable domain 1 (HV-1) and 2 (HV-2) (Bhat et al., 1991). New Opa protein variants constantly emerge, not only due to point mutations within $\mathrm{HV}-1$ and $\mathrm{HV}-2$ but also due to modular exchange of domains between different Opa proteins (Hobbs et al., 1994). Interestingly, the interaction of Opa proteins with different receptors on human cells has also been pinned down to the HV-1 and HV-2 regions (see below), posing the puzzling question of specific receptor recognition in the context of these sequence alterations.

Work since 1995 has identified the cellular receptors targeted by various Opa proteins. Historically, the first cellular receptor characterized was found to belong to the family of heparansulfate proteoglycans (HSPGs), highly glycosylated proteins that occur in transmembrane (syndecans) or glycosylphosphatidylinositol-anchored (glypicans) forms (David, 1993). 
Initially, it was observed that heparin addition or heparinase treatment abolishes gonococcal adhesion to different epithelial cells and that hamster cell lines deficient in heparansulfate biosynthesis were poorly recognized by the respective opaque gonococcal variants (Chen et al., 1995; van Putten and Paul, 1995). Additional investigations have revealed that both syndecan-1 and syndecan-4 can support not only the binding but also the internalization of Opa ${ }_{\mathrm{HSPG}}$-expressing gonococci (Freissler et al., 2000). So far, only a limited

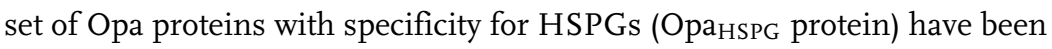
identified, including OpaA/Opa 30 of gonococcal strain MS11 and Opa 27.5 of strain VP1 (Kupsch et al., 1993; van Putten and Paul, 1995). Furthermore, Opa proteins with this type of specificity have not been described in meningococci.

On the bacterial side, HSPG binding has been mapped to the hypervariable extracellular loops of Opa proteins. From mutagenesis studies, it appears that a series of positively charged amino acids in HV-1 and HV-2 is critical in order to mediate the association with the HSPGs that have a high negative charge (Bos et al., 2002). Interestingly, HSPG-specific Opa proteins have additional binding capabilities. Depending on the cell line used, an increased Opa $a_{\text {HSP }}$ protein-triggered invasion has been observed in the presence of serum (Gomez-Duarte et al., 1997). Further analysis has suggested that $\mathrm{Opa}_{\mathrm{HSPG}}$ proteins also bind to the serum proteins vitronectin and fibronectin and, in a manner similar to the above described staphylococcal FnBPs, can therefore mediate an indirect engagement of host cell integrins (Dehio et al., 1998; van Putten et al., 1998). Again, indirect binding and clustering of integrins allow enhanced internalization by human non-professional phagocytes. As integrins and also syndecans are found ubiquitously on most

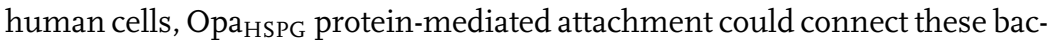
teria with numerous cell types during the infection. Whereas a few Opa proteins with binding specificity for HSPGs have been characterized, Opa ${ }_{\text {HSPG }}$ proteins represent only a minor fraction of the total Opa protein repertoire of most characterized strains.

In contrast, the majority of the currently characterized meningococcal and gonococcal Opa proteins display binding specificity for human surface receptors of the carcinoembryonic antigen (CEA)-related cell-adhesion molecule (CEACAM) family (Opa ${ }_{\mathrm{CEA}}$ proteins). Prototypes of this group of Opa proteins comprise, for example, $\mathrm{Opa}_{52}$ of gonococcal strain MS11 and $\mathrm{Opa}_{132}$ of meningococcal strain C751 (for Opa protein nomenclature,

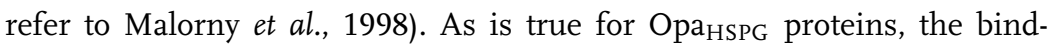
ing sites for CEACAMs reside within the hypervariable loops of CEACAMspecific Opa proteins. So far, no consensus motif has been elucidated that would predict the capabilities of a given Opa protein to bind to CEACAMs 
(Bos et al., 2002). What is more, the combination of hypervariable loops from

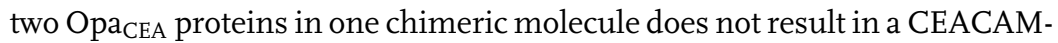
binding protein (Bos et al., 2002). These results are in line with the idea that CEACAM recognition is based on a three-dimensional structure created by the proper combination of two complementary hypervariable loops of Opa proteins. It is interesting to note that shuffling of hypervariable loops derived from CEACAM-binding Opa proteins in some cases created chimeric Opa proteins with a novel specificity for HSPGs (Bos et al., 2002). These striking observations suggest that HSPG recognition by Opa proteins might be a side product of the ongoing evolution and optimization of Opa $\mathrm{CEA}_{\mathrm{C}}$ molecules.

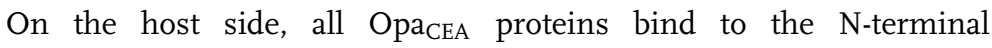
immunoglobulin variable-like $\left(\operatorname{Ig}_{\mathrm{v}}\right)$ domain characteristic for CEACAMs. Although this N-terminal domain is highly conserved among CEACAMs, opaque Neisseria have been found to recognize only four of the seven CEACAMs expressed by their human host, namely CEACAM1, CEACAM3, CEA (the product of the ceacam 5 gene), and CEACAM6. Common to these CEACAM family members is the presence of the CD66 epitope in their $\mathrm{N}$ terminal domain; therefore, these proteins have formerly been designated CD66a (CEACAM1), CD66c (CEACAM6), CD66d (CEACAM3), and CD66e (CEA). In contrast, the additional family members CEACAM4, CEACAM7, and CEACAM8 (CD66b) are not bound by any Opa far (Popp et al., 1999). On the basis of Opa ${ }_{\text {CEA }}$-binding and non-binding CEACAM N-terminal domains, a number of receptor chimeras and mutants

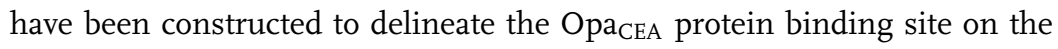
non-glycosylated C'CFG-face of the immunoglobulin domain fold (reviewed in Billker et al., 2000). The crystal structure of murine CEACAM1, the only member of this glycoprotein family found in mice, has revealed a characteristic surface-exposed loop coordinated by Tyr-34 within the $\mathrm{C}^{\prime} \mathrm{CFG}$ face (Tan et al., 2002). It is assumed that a similar prominent surface extension

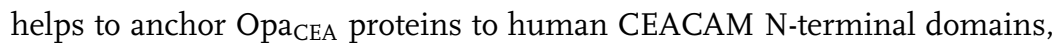
as mutagenesis of the corresponding Tyr-34 residue in human CEACAM1

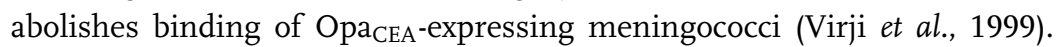
It is interesting to point out that in mice, CEACAM1 serves as the cellular receptor for mouse hepatitis virus (MHV), and MHV binding also takes place at the N-terminal Ig $_{\mathrm{v}}$-like domain (Dveksler et al., 1993). Importantly, the genetic ablation of the murine CEACAM1 N-terminal domain from the mouse genome has resulted in animals resistant to $\mathrm{MHV}$ infection, pointing towards the essential role that the pathogen-CEACAM interaction plays in this system (Blau et al., 2001). 
Although the physiologic function of CEACAMs in vivo is not understood completely, some family members are known to mediate cell-cell adhesion in vitro via both homotypic (CEACAM1, CEA, CEACAM6) and/or heterotypic (CEA-CEACAM6 and CEACAM6-CEACAM8) interactions (Benchimol et al., 1989; Oikawa et al., 1991). CEACAM1 and CEACAM6 on neutrophils are also involved in the adherence of activated neutrophils to cytokine-activated endothelial cells, both directly through their ability to present the sialylated Lewis ${ }^{x}$ antigen to E-selectin and indirectly by the CEACAM6-stimulated activation of CD18 integrins (Kuijpers et al., 1992). Interestingly, CEACAM1 is also implicated in hepatic uptake of insulin, demonstrating that CEACAMs could participate in internalization processes under physiological conditions (Poy, Ruch et al., 2002; Poy, Yang et al., 2002).

An important aspect of CEACAM biology is the fact that several CEACAM family members can be expressed by epithelial cells, where they are usually located at the apical membrane of the polarized epithelium (Hammarstrom, 1999). This has important implications with respect to their role as bacterial receptors, as they are prominently exposed on mucosal surfaces and, therefore, accessible for incoming microbes. Furthermore, CEACAMs have been linked to signal transduction into the cell, and some isoforms seem to be connected to the intracellular cytoskeleton (Obrink, 1997).

Most work in this regard has focused on CEACAM1, the most widely expressed CEACAM family member. CEACAM1 is not only abundantly expressed on epithelia (including stomach, colon, kidney, gall bladder, liver, urinary bladder, prostate, cervix, and endometrium), sweat and sebaceous gland cells, and endothelia but also found on leukocytes such as granulocytes and B- and T-cells (Hammarstrom, 1999). In addition, CEACAM1 homologs are found in non-primate species such as mouse and rat, enabling better experimental access. In epithelial cells, CEACAM1 localizes to cell-cell contacts and is associated with the actin cytoskeleton under the control of Rhofamily GTPases (Sadekova et al., 2000). Moreover, the isolated cytoplasmic domain of CEACAM1 binds to actin and tropomyosin in vitro (Schumann et al., 2001), supporting the view that CEACAM1 is connected directly to the cytoskeleton and plays a role in maintaining tissue integrity. Other groups report a direct association between the cytoplasmic domain of CEACAM1 and $\beta 3$ integrins, indicating that there could also be an indirect linkage, via integrins, between CEACAMs and the actin cytoskeleton (Brummer et al., 2001). However, pharmacological inhibitors of the actin cytoskeleton do not seem to influence CEACAM1- and CEACAM6-mediated uptake of opaque bacteria (Billker et al., 2002; McCaw et al., 2004). 
Binding of Opa $a_{\text {CEA }}$ proteins to members of the CEACAM family is sufficient to induce the internalization of the bacteria into several cell types in vitro. Several CEACAMs are expressed on human granulocytes, where in particular CEACAM3 promotes an efficient opsonin-independent uptake of CEACAMbinding bacteria (Schmitter et al., 2004). With regard to epithelial cells, CEACAM1, CEA, and CEACAM6 are often found to be coexpressed. In polarized T84 epithelial cell monolayers, CEA, CEACAM1, and CEACAM6 are transported apically, where they mediate invasion and subsequent transcytosis of

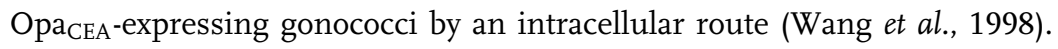
In primary human umbilical vein endothelial cells (HUVECs) and in certain epithelial cells such as cells derived from human ovary, CEACAM1 expression is low in resting cells. However, CEACAM1 expression can be dramatically induced by infection with pathogenic Neisseriae, leading in turn to increased adherence and internalization of Opa-positive variants (Muenzner et al., 2001, 2002).

It is conceivable, therefore, that Opa-mediated binding to CEACAMs is an important mechanism that allows the pathogens to successfully colonize human mucosal surfaces. According to this hypothesis, Opa expression by the bacteria and presence or upregulation of CEACAMs on epithelia or endothelia upon contact with the microorganisms would act as a switch to facilitate bacterial colonization and potentially also to enhance the passage of pathogenic Neisseriae through epithelial (and endothelial) barriers. It is important to point out that several other Gram-negative human-specific pathogens that share the same ecological niche and cause a similar spectrum of diseases as gonococci and meningococci have been found to possess CEACAM-binding adhesins. In an example of convergent evolution, typeable and non-typeable Haemophilus influenzae, $H$. influenzae biogroup aegyptius, and Moraxella catarrhalis have elaborated diverse surface antigens to engage CEACAMs (Hill and Virji, 2003; Virji et al., 2000). For example, H. influenzae contacts CEACAM family members by the outer-membrane protein P5, whereas M. catarrhalis employs the UspA1 antigen (Hill and Virji, 2003; Hill et al., 2001). These findings imply that there must be some major advantage for bacteria colonizing the human mucosa to specifically target members of the CEACAM family.

Novel insight suggests that in addition to providing a tight molecular anchor on the apical side of human epithelia, CEACAM recognition might serve an even more elaborate function in support of mucosal colonization. More specifically, CEACAM engagement by human pathogens might be a means to blunt an innate defense mechanism of stratified and squamous epithelial tissues, namely the exfoliation of superficial cells. 


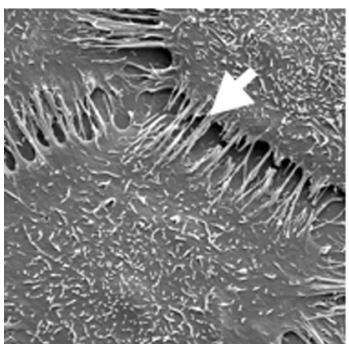

(a)

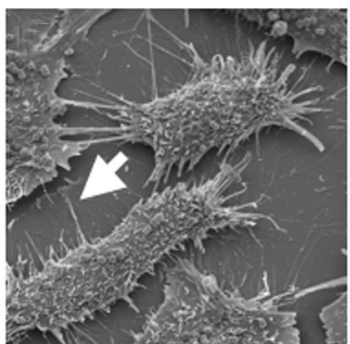

(b)

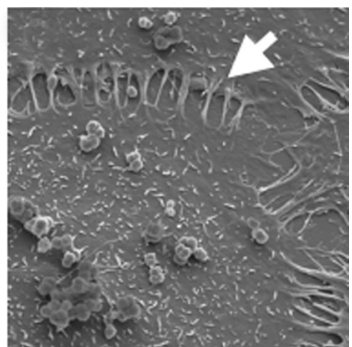

(c)

Figure 6.1 Epithelial cells infected with carcinoembryonic antigen-related cell-adhesion molecule (CEACAM)-binding Neisseria gonorrhoeae do not detach after prolonged infection. Confluent monolayers of human cervix epithelial cell line (ME-180) grown on collagen-coated surfaces were left (a) uninfected, or (b) infected for $14 \mathrm{~h}$ with piliated non-opaque $N$. gonorrhoeae (NgoP+), or (c) infected with non-piliated Opa ${ }_{\mathrm{CEA}}$-expressing $N$. gonorrhoeae (Ngo Opa ${ }_{\text {CEA }}$ ). Cultures were fixed in situ and analyzed by scanning electron microscopy. Whereas uninfected monolayers display well-spread epithelial cells with numerous cell-cell contacts (a; arrow), ME-180 cells infected with piliated gonococci lose cell-cell contacts (B; arrow), round up and detach from the extracellular matrix surface, consistent with bacteria-induced exfoliation. In contrast, epithelial cells infected with CEACAM-binding gonococci (an OpaCEA-expressing strain) stay attached to the matrix, although cell-cell contacts are diminished (c; arrow). Figures courtesy of M. Rohda, GBF, Braunschweig, Germany.

Importantly, different non-opaque gonococcal variants cause detachment of epithelial cells after prolonged infection in culture, and such an exfoliation of urethral cells has been reported to occur during gonorrhea in vivo (Apicella et al., 1996; Evans, 1977; Melly et al., 1981; Mosleh et al., 1997; Ward et al., 1974). In striking contrast, prolonged infection with CEACAM-binding gonococci does not result in epithelial exfoliation (Muenzner et al., 2005). This process can be documented by scanning electron microscopy, whereupon infection of a confluent monolayer of a human cervix epithelial cell line with piliated non-opaque gonococci, a reduction in cell-cell contacts, and the rounding and detachment of infected epithelial cells can be observed clearly (Figure 6.1). However, when these cervix epithelial cells that endogenously express CEACAM family members are

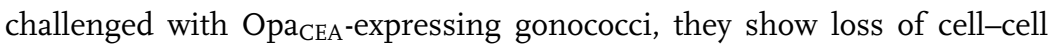
contacts but still remain attached to the underlying extracellular matrix (Figure 6.1). The lack of detachment was attributed to a dramatically increased adhesive property of cells infected with opaque Neisseria (Muenzner et al., 2005). 
By microarray-based gene expression analysis and further functional studies, the enhanced adhesive properties of the infected cells could be pinned down to the de novo expression of CD105 following CEACAM engagement. Importantly, upregulation of CD105 occurs in response to a number of CEACAM-binding human pathogens, including $N$. gonorrhoeae, N. meningitidis, $H$. influenzae, and $M$. catarrhalis, and is sufficient to promote increased cell adhesion (Muenzner et al., 2005). Therefore, it is conceivable that pathogen-initiated CEACAM stimulation, the stimulated expression of CD105, and the ensuing enhanced cell-matrix adhesion are central events that counteract the exfoliation of infected epithelial cells in vivo and, therefore, facilitate the colonization of the human mucosa by CEACAM-binding bacteria. In light of these results, it is tempting to speculate that the prevention of epithelial detachment attained through CEACAM binding is the major evolutionary driving force behind the appearance of distinct CEACAM-directed adhesins in several bacterial species colonizing the human mucosa.

\section{CONCLUSIONS}

Pathogen-epithelial cell recognition and the tight attachment of microorganisms to this host cell type are of fundamental importance in major infectious diseases. Since the initial discoveries of the first fimbrial and afimbrial adhesins, work by numerous research groups has led to the identification of numerous adhesin-receptor pairs, and these molecular investigations still yield exciting and often surprising insight. Fimbrial adhesins have long been recognized as critical bacterial surface structures mediating the initial contact between the prokaryotic and eukaryotic worlds. In addition, a large variety of afimbrial adhesins provides pathogens with an additional arsenal to interact intimately with target cells and to trigger specific responses upon receptor engagement. The more we learn about the intricate molecular communication taking place at the bacterial-epithelial cell interface, the more it is becoming evident that receptor targeting by bacterial adhesins has effects beyond the attachment to the host cell surface. The coming years will witness an increased appreciation and understanding of such post-adhesion events that modulate and shape the infection process.

\section{ACKNOWLEDGMENTS}

I thank the members of my laboratory for stimulating discussions during the writing process and the Bundesministerium für Bildung und Forschung 
as well as the Deutsche Forschungsgemeinschaft for support of our research in this area.

\section{REFERENCES}

Agerer, F., Michel, A., Ohlsen, K., and Hauck, C. R. (2003). Integrin-mediated invasion of Staphylococcus aureus into human cells requires Src family protein tyrosine kinases. J. Biol. Chem. 278, 42 524-42531.

Agerer, F., Lux, S., Michel, A., et al. (2005). Cellular invasion by Staphylococcus aureus reveals a functional link between focal adhesion kinase and cortactin in integrin-mediated internalisation. J. Cell Sci. 118, 2189-2200.

Apicella, M. A., Ketterer, M., Lee, F. K. N., et al. (1996). The pathogenesis of gonococcal urethritis in men: confocal and immunoelectron microscopic analysis of urethral exsudates from men infected with Neisseria gonorrhoeae. J. Infect. Dis. 173, 636-646.

Ayala, B. P., Vasquez, B., Clary, S., et al. (2001). The pilus-induced Ca2+ flux triggers lysosome exocytosis and increases the amount of Lamp1 accessible to Neisseria IgA1 protease. Cell. Microbiol. 3, 265-275.

Beachey, E. H. (1981). Bacterial adherence: adhesin-receptor interactions mediating the attachment of bacteria to mucosal surface. J. Infect. Dis. 143, 325345 .

Benchimol, S., Fuks, A., Jothy, S., et al. (1989). Carcinoembryonic antigen, a human tumor marker, functions as an intercellular adhesion molecule. Cell 57, 327-334.

Bhat, K. S., Gibbs, C. P., Barrera, O., et al. (1991). The opacity proteins of Neisseria gonorrhoeae strain MS11 are encoded by a family of 11 complete genes. Mol. Microbiol. 5, 1889-1901. [Published erratum appears in Mol. Microbiol. 1992, 6, 1073-1076.]

Billker, O., Popp, A., Gray-Owen, S. D., and Meyer, T. F. (2000). The structural basis of CEACAM-receptor targeting by neisserial Opa proteins. Trends Microbiol. 8, 258-260.

Billker, O., Popp, A., Brinkmann, V., et al. (2002). Distinct mechanisms of internalization of Neisseria gonorrhoeae by members of the CEACAM receptor family involving Rac1- and Cdc42- dependent and -independent pathways. EMBO J. 21, 560-571.

Blau, D. M., Turbide, C., Tremblay, M., et al. (2001). Targeted disruption of the Ceacam1 (MHVR) gene leads to reduced susceptibility of mice to mouse hepatitis virus infection. J. Virol. 75, 8173-8186.

Bos, M. P., Kao, D., Hogan, D. M., Grant, C. C., and Belland, R. J. (2002) Carcinoembryonic antigen family receptor recognition by gonococcal Opa proteins 
requires distinct combinations of hypervariable Opa protein domains. Infect. Immun. 70, 1715-1723.

Bouckaert, J., Berglund, J., Schembri, M., et al. (2005). Receptor binding studies disclose a novel class of high-affinity inhibitors of the Escherichia coli FimH adhesin. Mol. Microbiol. 55, 441-455.

Brouillette, E., Grondin, G., Shkreta, L., Lacasse, P., and Talbot, B. G. (2003). In vivo and in vitro demonstration that Staphylococcus aureus is an intracellular pathogen in the presence or absence of fibronectin-binding proteins. Microb. Pathog. 35, 159-168.

Brummer, J., Ebrahimnejad, A., Flayeh, R., et al. (2001). cis Interaction of the cell adhesion molecule CEACAM1 with integrin beta(3). Am. J. Pathol. 159, 537-546.

Chen, T., Belland, R. J., Wilson, J., and Swanson, J. (1995). Adherence of pilusOpa+ gonococci to epithelial cells in vitro involves heparan sulfate. J. Exp. Med. 182, 511-517.

Clark, M. A., Hirst, B. H., and Jepson, M. A. (1998). M-cell surface beta1 integrin expression and invasin-mediated targeting of Yersinia pseudotuberculosis to mouse Peyer's patch M cells. Infect. Immun. 66, 1237-1243.

Cohen, M. S. and Cannon, J. G. (1999). Human experimentation with Neisseria gonorrhoeae: progress and goals. J. Infect. Dis. 179 (Suppl 2), S375-379.

Craig, L., Pique, M. E., and Tainer, J. A. (2004). Type IV pilus structure and bacterial pathogenicity. Nat. Rev. Microbiol. 2, 363-378.

David, G. (1993). Integral membrane heparan sulfate proteoglycans. FASEB J. 7, 1023-1030.

Deghmane, A. E., Giorgini, D., Larribe, M., Alonso, J. M., and Taha, M. K. (2002). Down-regulation of pili and capsule of Neisseria meningitidis upon contact with epithelial cells is mediated by CrgA regulatory protein. Mol. Microbiol. 43, 1555-1564.

Dehio, M., Gomez-Duarte, O. G., Dehio, C., and Meyer, T. F. (1998). Vitronectindependent invasion of epithelial cells by Neisseria gonorrhoeae involves alpha(v) integrin receptors. FEBS Lett. 424, 84-88.

De Jonge, M. I., Bos, M. P., Hamstra, H. J., et al. (2002). Conformational analysis of opacity proteins from Neisseria meningitidis. Eur. J. Biochem. 269, 52155223.

Dersch, P. and Isberg, R. R. (1999). A region of the Yersinia pseudotuberculosis invasin protein enhances integrin-mediated uptake into mammalian cells and promotes self-association. EMBO J. 18, 1199-1213.

Duguid, J. P. (1959). Fimbriae and adhesive properties in Klebsiella strains. J. Gen. Microbiol. 21, 271-286. 
Dveksler, G. S., Dieffenbach, C. W., Cardellichio, C. B., et al. (1993). Several members of the mouse carcinoembryonic antigen-related glycoprotein family are functional receptors for the coronavirus mouse hepatitis virus-A59. J. Virol. 67, 1-8.

Eden, C. S., Hanson, L. A., Jodal, U., Lindberg, U., and Akerlund, A. S. (1976). Variable adherence to normal human urinary-tract epithelial cells of Escherichia coli strains associated with various forms of urinary-tract infection. Lancet 1, 490-492.

Edwards, N. J., Monteiro, M. A., Faller, G., et al. (2000). Lewis X structures in the $\mathrm{O}$ antigen side-chain promote adhesion of Helicobacter pylori to the gastric epithelium. Mol. Microbiol. 35, 1530-1539.

Evans, B. A. (1977). Ultrastructural study of cervical gonorrhea. J. Infect. Dis. 136, 248-255.

Foster, T. J. and Hook, M. (1998). Surface protein adhesins of Staphylococcus aureus. Trends Microbiol. 6, 484-488.

Freissler, E., Meyer auf der Heyde, A., David, G., Meyer, T. F., and Dehio, C. (2000). Syndecan-1 and syndecan-4 can mediate the invasion of Opa $\mathrm{HSPG}^{-}$ expressing Neisseria gonorrhoeae into epithelial cells. Cell. Microbiol. 2, 69-82.

Gomez-Duarte, O. G., Dehio, M., Guzman, C. A., et al. (1997). Binding of vitronectin to Opa-expressing Neisseria gonorrhoeae mediates invasion of $\mathrm{HeLa}$ cells. Infect. Immun. 65, 3857-3866.

Guyot, G. (1908). Über die bakterielle Adhäsion. Zentralbl. Bakteriol. 46, 640-653.

Hammarstrom, S. (1999). The carcinoembryonic antigen (CEA) family: structures, suggested functions and expression in normal and malignant tissues. Semin. Cancer Biol. 9, 67-81.

Helaine, S., Carbonnelle, E., Prouvensier, L., et al. (2005). PilX, a pilus-associated protein essential for bacterial aggregation, is a key to pilus-facilitated attachment of Neisseria meningitidis to human cells. Mol. Microbiol. 55, 65-77.

Hill, D. J. and Virji, M. (2003). A novel cell-binding mechanism of Moraxella catarrhalis ubiquitous surface protein UspA: specific targeting of the $\mathrm{N}$ domain of carcinoembryonic antigen-related cell adhesion molecules by UspA1. Mol. Microbiol. 48, 117-129.

Hill, D. J., Toleman, M. A., Evans, D. J., et al. (2001). The variable P5 proteins of typeable and non-typeable Haemophilus influenzae target human CEACAM1. Mol. Microbiol. 39, 850-862.

Hobbs, M. M., Seiler, A., Achtmann, M., and Cannon, J. G. (1994). Microevolution within a clonal population of pathogenic bacteria: recombination, gene duplication and horizontal genetic exchange in the opa gene family of Neisseria meningitidis. Mol. Microbiol. 12, 171-180. 
Hoffmann, I., Eugene, E., Nassif, X., Couraud, P. O., and Bourdoulous, S. (2001). Activation of ErbB2 receptor tyrosine kinase supports invasion of endothelial cells by Neisseria meningitidis. J. Cell Biol. 155, 133-143.

Ilic, D., Kovacic, B., Johkura, K., et al. (2004). FAK promotes organization of fibronectin matrix and fibrillar adhesions. J. Cell Sci. 117, 177-187.

Isberg, R. R. and Leong, J. M. (1990). Multiple $b_{1}$ chain integrins are receptors for invasin, a protein that promotes bacterial penetration into mammalian cells. Cell 60, 861-871.

Isberg, R. R., Hamburger, Z., and Dersch, P. (2000). Signaling and invasinpromoted uptake via integrin receptors. Microbes Infect. 2, 793-801.

Jerse, A. E., Cohen, M. S., Drown, P. M., et al. (1994). Multiple gonococcal opacity proteins are expressed during experimental urethral infection in the male. $J$. Exp. Med. 179, 911-920.

Johansson, L., Rytkonen, A., Bergman, P., et al. (2003). CD46 in meningococcal disease. Science 301, 373-375.

Jonson, A. B., Normark, S., and Rhen, M. (2005). Fimbriae, pili, flagella and bacterial virulence. Contrib. Microbiol. 12, 67-89.

Kallstrom, H., Liszewski, M. K., Atkinson, J. P., and Jonsson, A. B. (1997). Membrane cofactor protein (MCP or CD46) is a cellular pilus receptor for pathogenic Neisseria. Mol. Microbiol. 25, 639-647.

Kallstrom, H., Islam, M. S., Berggren, P.-O., and Jonsson, A.-B. (1998). Cell signaling by the type IV pili of pathogenic Neisseria. J. Biol. Chem. 273, $21777-$ 21782.

Kau, A. L., Hunstad, D. A., and Hultgren, S. J. (2005). Interaction of uropathogenic Escherichia coli with host uroepithelium. Curr. Opin. Microbiol. 8, 5459.

Kellogg, D. S., Peacock, W. L., Deacon, W. E., Brown, L., and Pirkle, C. I. (1963). Neisseria gonorrhoeae: I. Virulence linked to clonal variation. J. Bacteriol. 85, 1274-1279.

Kirchner, M. and Meyer, T. F. (2005). The PilC adhesin of the Neisseria type IV pilus: binding specificities and new insights into the nature of the host cell receptor. Mol. Microbiol. 56, 945-957.

Kirchner, M., Heuer, D., and Meyer, T. F. (2005). CD46-independent binding of neisserial type IV pili and the major pilus adhesin, PilC, to human epithelial cells. Infect. Immun. 73, 3072-3082.

Kuijpers, T. W., Hoogerwerf, M., van der Laan, L. J., et al. (1992). CD66 nonspecific cross-reacting antigens are involved in neutrophil adherence to cytokineactivated endothelial cells. J. Cell Biol. 118, 457-466.

Kupsch, E. M., Knepper, B., Kuroki, T., Heuer, I., and Meyer, T. F. (1993). Variable opacity (Opa) outer membrane proteins account for the cell tropisms 
displayed by Neisseria gonorrhoeae for human leukocytes and epithelial cells. EMBO J. 12, 641-650.

Kuypers, J. M. and Proctor, R. A. (1989). Reduced adherence to traumatized rat heart valves by a low-fibronectin-binding mutant of Staphylococcus aureus. Infect. Immun. 57, 2306-2312.

Lecuit, M., Dramsi, S., Gottardi, C., et al. (1999). A single amino acid in E-cadherin responsible for host specificity towards the human pathogen Listeria monocytogenes. EMBO J. 18, 3956-3963.

Lecuit, M., Vandormael-Pournin, S., Lefort, J., et al. (2001). A transgenic model for listeriosis: role of internalin in crossing the intestinal barrier. Science 292, 1722-1725.

Lee, S. W., Bonnah, R. A., Higashi, D. L., et al. (2002). CD46 is phosphorylated at tyrosine 354 upon infection of epithelial cells by Neisseria gonorrhoeae. J. Cell Biol. 156, 951-957.

Maier, B., Potter, L., So, M., et al. (2002). Single pilus motor forces exceed 100 pN. Proc. Natl. Acad. Sci. U. S. A. 99, 16 012-16017.

Malorny, B., Morelli, G., Kusecek, B., Kolberg, J., and Achtman, M. (1998). Sequence diversity, predicted two-dimensional protein structure, and epitope mapping of neisserial Opa proteins. J. Bacteriol. 180, 1323-1330.

Marra, A. and Isberg, R. R. (1997). Invasin-dependent and invasin-independent pathways for translocation of Yersinia pseudotuberculosis across the Peyer's patch intestinal epithelium. Infect. Immun. 65, 3412-3421.

McCaw, S. E., Liao, E. H., and Gray-Owen, S. D. (2004). Engulfment of Neisseria gonorrhoeae: revealing distinct processes of bacterial entry by individual carcinoembryonic antigen-related cellular adhesion molecule family receptors. Infect. Immun. 72, 2742-2752.

McCormick, B. A. (2003). The use of transepithelial models to examine hostpathogen interactions. Curr. Opin. Microbiol. 6, 77-81.

McNeish, A. S., Turner, P., Fleming, J., and Evans, N. (1975). Mucosal adherence of human enteropathogenic Escherichia coli. Lancet 2, 946-948.

McNiven, M. A., Kim, L., Krueger, E. W., et al. (2000). Regulated interactions between dynamin and the actin-binding protein cortactin modulate cell shape. J. Cell Biol. 151, 187-198.

Melly, M. A., Gregg, C. R., and McGee, Z. A. (1981). Studies of toxicity of Neisseria gonorrhoeae for human fallopian tube mucosa. J. Infect. Dis. 143, 423-431.

Mengaud, J., Ohayon, H., Gounon, P., Mege, R. M., and Cossart, P. (1996). Ecadherin is the receptor for internalin, a surface protein required for entry of L. monocytogenes into epithelial cells. Cell 84, 923-932.

Menzies, B. E. (2003). The role of fibronectin binding proteins in the pathogenesis of Staphylococcus aureus infections. Curr. Opin. Infect. Dis. 16, 225-229. 
Menzies, B. E., Kourteva, Y., Kaiser, A. B., and Kernodle, D. S. (2002). Inhibition of staphylococcal wound infection and potentiation of antibiotic prophylaxis by a recombinant fragment of the fibronectin-binding protein of Staphylococcus aureus. J. Infect. Dis. 185, 937-943.

Merz, A. J., So, M., and Sheetz, M. P. (2000). Pilus retraction powers bacterial twitching motility. Nature 407, 98-102.

Meyer, T. F., Gibbs, C. P., and Haas, R. (1990). Variation and control of protein expression in Neisseria. Annu. Rev. Microbiol. 44, 451-477.

Morand, P. C., Tattevin, P., Eugene, E., Beretti, J.-L., and Nassif, X. (2001). The adhesive property of the type IV pilus-associated component PilC1 of pathogenic Neisseria is supported by the conformational structure of the Nterminal part of the molecule. Mol. Microbiol. 40, 846-856.

Morand, P. C., Bille, E., Morelle, S., et al. (2004). Type IV pilus retraction in pathogenic Neisseria is regulated by the PilC proteins. EMBO J. 23, 20092017.

Mosleh, I. M., Boxberger, H. J., Sessler, M. J., and Meyer, T. F. (1997). Experimental infection of native human ureteral tissue with Neisseria gonorrhoeae: adhesion, invasion, intracellular fate, exocytosis, and passage through a stratified epithelium. Infect. Immun. 65, 3391-3398.

Muenzner, P., Naumann, M., Meyer, T. F., and Gray-Owen, S. D. (2001). Pathogenic Neisseria trigger expression of their carcinoembryonic antigenrelated cellular adhesion molecule 1 (CEACAM1; previously CD66a) receptor on primary endothelial cells by activating the immediate early response transcription factor, nuclear factor-kappa B. J. Biol. Chem. 276, $24331-$ 24340.

Muenzner, P., Billker, O., Meyer, T. F., and Naumann, M. (2002). Nuclear factor$\mathrm{kB}$ directs CEACAM1 receptor expression in Neisseria gonorrhoeae-infected epithelial cells. J. Biol. Chem. 277, 7438-7446.

Muenzner, P., Rohde, M., Kneitz, S., and Hauck, C. R. (2005). CEACAM engagement by human pathogens enhances cell adhesion and counteracts bacteriainduced detachment of epithelial cells. J. Cell Biol. 170, 825-836.

Obrink, B. (1997). CEA adhesion molecules: multifunctional proteins with signalregulatory properties. Curr. Opin. Cell Biol. 9, 616-626.

Ofek, I., Beachey, E. H., Jefferson, W., and Campbell, G. L. (1975). Cell membranebinding properties of group A streptococcal lipoteichoic acid. J. Exp. Med.141, 990-1003.

Oikawa, S., Inuzuka, C., Kuroki, M., et al. (1991). A specific heterotypic cell adhesion activity between members of carcinoembryonic antigen family, W272 and NCA, is mediated by N-domains. J. Biol. Chem. 266, 79958001. 
Old, D. C. (1972). Inhibition of the interaction between fimbrial haemagglutinins and erythrocytes by D-mannose and other carbohydrates. J. Gen. Microbiol. 71, 149-157.

Ozeri, V., Rosenshine, I., Mosher, D. F., Fassler, R., and Hanski, E. (1998). Roles of integrins and fibronectin in the entry of Streptococcus pyogenes into cells via protein F1. Mol. Microbiol. 30, 625-637.

Paradis, S. E., Dubreuil, J. D., Gottschalk, M., Archambault, M., and Jacques, M. (1999). Inhibition of adherence of Actinobacillus pleuropneumoniae to porcine respiratory tract cells by monoclonal antibodies directed against LPS and partial characterization of the LPS receptors. Curr. Microbiol. 39, 313-320.

Patti, J. M., Allen, B. L., McGavin, M. J., and Hook, M. (1994). MSCRAMMmediated adherence of microorganisms to host tissues. Annu. Rev. Microbiol. 48, 585-617.

Pizarro-Cerda, J., Sousa, S., and Cossart, P. (2004). Exploitation of host cell cytoskeleton and signalling during Listeria monocytogenes entry into mammalian cells. C. R. Biol. 327, 115-123.

Popp, A., Dehio, C., Grunert, F., Meyer, T. F., and Gray-Owen, S. D. (1999). Molecular analysis of neisserial Opa protein interactions with the CEA family of receptors: identification of determinants contributing to the differential specificities of binding. Cell. Microbiol. 1, 169-181.

Poy, M. N., Ruch, R. J., Fernstrom, M. A., Okabayashi, Y., and Najjar, S. M. (2002). Shc and CEACAM1 interact to regulate the mitogenic action of insulin. J. Biol. Chem. 277, 1076-1084.

Poy, M. N., Yang, Y., Rezaei, K., et al. (2002). CEACAM1 regulates insulin clearance in liver. Nat. Genet. 19, 19.

Pujol, C., Eugene, E., de Saint Martin, L., and Nassif, X. (1997). Interaction of Neisseria meningitidis with a polarized monolayer of epithelial cells. Infect. Immun. 65, 4836-4842.

Punsalang, A. P. Jr and Sawyer, W. D. (1973). Role of pili in the virulence of Neisseria gonorrhoeae. Infect. Immun. 8, 255-263.

Rudel, T., Scheuerpflug, I., and Meyer, T. F. (1995). Neisseria PilC protein identified as type-4 pilus-tip located adhesin. Nature 373, 357-359.

Ryll, R. R., Rudel, T., Scheuerpflug, I., Barten, R., and Meyer, T. F. (1997). PilC of Neisseria meningitidis is involved in class II pilus formation and restores pilus assembly, natural transformation competence and adherence to epithelial cells in PilC-deficient gonococci. Mol. Microbiol. 23, 879-892.

Sadekova, S., Lamarche-Vane, N., Li, X., and Beauchemin, N. (2000). The CEACAM1-L glycoprotein associates with the actin cytoskeleton and localizes to cell-cell contact through activation of Rho-like GTPases. Mol. Biol. Cell 11, 65-77. 
Sauer, F. G., Remaut, H., Hultgren, S. J., and Waksman, G. (2004). Fiber assembly by the chaperone-usher pathway. Biochim. Biophys. Acta 1694, 259-267.

Scheuerpflug, I., Rudel, T., Ryll, R., Pandit, J., and Meyer, T. F. (1999). Roles of PilC and PilE proteins in pilus-mediated adherence of Neisseria gonorrhoeae and Neisseria meningitidis to human erythrocytes and endothelial and epithelial cells. Infect. Immun. 67, 834-843.

Schmitter, T., Agerer, F., Peterson, L., Muenzner, P., and Hauck, C. R. (2004). Granulocyte CEACAM3 is a phagocytic receptor of the innate immune system that mediates recognition and elimination of human-specific pathogens. J. Exp. Med. 199, 35-46.

Schubert, W.-D., Urbanke, C., Ziehm, T., et al. (2002). Structure of internalin, a major invasion protein of Listeria monocytogenes, in complex with its human receptor E-cadherin. Cell 111, 825.

Schumann, D., Chen, C. J., Kaplan, B., and Shively, J. E. (2001). Carcinoembryonic antigen cell adhesion molecule 1 directly associates with cytoskeleton proteins actin and tropomyosin. J. Biol. Chem. 276, 47 421-47 433.

Schwarzbauer, J. E. and Sechler, J. L. (1999). Fibronectin fibrillogenesis: a paradigm for extracellular matrix assembly. Curr. Opin. Cell Biol.11, 622-627.

Schwarz-Linek, U., Werner, J. M., Pickford, A. R., et al. (2003). Pathogenic bacteria attach to human fibronectin through a tandem beta-zipper. Nature 423, 177181.

Selbach, M. and Backert, S. (2005). Cortactin: an Achilles' heel of the actin cytoskeleton targeted by pathogens. Trends Microbiol. 13, 181-189.

Simonet, M., Riot, B., Fortineau, N., and Berche, P. (1996). Invasin production by Yersinia pestis is abolished by insertion of an IS200-like element within the inv gene. Infect. Immun. 64, 375-379.

Sinha, B., Francois, P. P., Nusse, O., et al. (1999). Fibronectin-binding protein acts as Staphylococcus aureus invasin via fibronectin bridging to integrin alpha5beta1. Cell. Microbiol. 1, 101-117.

Stern, A. and Meyer, T. F. (1987). Common mechanism controlling phase and antigenic variation in pathogenic neisseriae. Mol. Microbiol. 1, 5-12.

Stern, A., Brown, M., Nickel, P., and Meyer, T. F. (1986). Opacity genes in Neisseria gonorrhoeae: control of phase and antigenic variation. Cell 47, 61-71.

Swanson, J., Barrera, O., Sola, J., and Boslego, J. (1988). Expression of outer membrane protein II by gonococci in experimental gonorrhea. J. Exp. Med. 168, 2121-2129.

Tan, K., Zelus, B. D., Meijers, R., et al. (2002). Crystal structure of murine sCEACAM1a[1,4]: a coronavirus receptor in the CEA family. EMBO J. 21, 2076-2086. 
Tobiason, D. M. and Seifert, H. S. (2001). Inverse relationship between pilusmediated gonococcal adherence and surface expression of the pilus receptor, CD46. Microbiology 147, 2333-2340.

Ton-That, H. and Schneewind, O. (2004). Assembly of pili in Gram-positive bacteria. Trends Microbiol. 12, 228-234.

Vandeputte-Rutten, L., Bos, M. P., Tommassen, J., and Gros, P. (2003). Crystal structure of neisserial surface protein A (NspA), a conserved outer membrane protein with vaccine potential. J. Biol. Chem. 278, 24825-24830.

Van Putten, J. P. and Paul, S. M. (1995). Binding of syndecan-like cell surface proteoglycan receptors is required for Neisseria gonorrhoeae entry into human mucosal cells. EMBO J. 14, 2144-2154.

Van Putten, J. P., Duensing, T. D., and Cole, R. L. (1998). Entry of OpaA+ gonococci into HEp-2 cells requires concerted action of glycosaminoglycans, fibronectin and integrin receptors. Mol. Microbiol. 29, 369-379.

Virji, M., Evans, D., Hadfield, A., et al. (1999). Critical determinants of host receptor targeting by Neisseria meningitidis and Neisseria gonorrhoeae: identification of Opa adhesiotopes on the N-domain of CD66 molecules. Mol. Microbiol. 34, 538-551.

Virji, M., Evans, D., Griffith, J., et al. (2000). Carcinoembryonic antigens are targeted by diverse strains of typable and non-typable Haemophilus influenzae. Mol. Microbiol. 36, 784-795.

Volberg, T., Romer, L., Zamir, E., and Geiger, B. (2001). pp60(c-src) and related tyrosine kinases: a role in the assembly and reorganization of matrix adhesions. J. Cell Sci. 114, 2279-2289.

Wang, J., Gray-Owen, S. D., Knorre, A., Meyer, T. F., and Dehio, C. (1998). Opa binding to cellular CD66 receptors mediates the transcellular traversal of Neisseria gonorrhoeae across polarized T84 epithelial cell monolayers. Mol. Microbiol. 30, 657-671.

Ward, M. E., Watt, P. J., and Robertson, J. N. (1974). The human fallopian tube: a laboratory model for gonococcal infection. J. Infect. Dis. 129, 650-659.

Weidenmaier, C., Kokai-Kun, J. F., Kristian, S. A., et al. (2004). Role of teichoic acids in Staphylococcus aureus nasal colonization, a major risk factor in nosocomial infections. Nat. Med. 10, 243-245.

Zaidel-Bar, R., Cohen, M., Addadi, L., and Geiger, B. (2004). Hierarchical assembly of cell-matrix adhesion complexes. Biochem. Soc. Trans. 32, 416-420.

Zamir, E. and Geiger, B. (2001). Molecular complexity and dynamics of cell-matrix adhesions. J. Cell Sci. 114, 3583-3590.

Zamir, E., Katz, M., Posen, Y., et al. (2000). Dynamics and segregation of cellmatrix adhesions in cultured fibroblasts. Nat. Cell Biol. 2, 191-196. 